\title{
A CASE REPORT OF ANTIPHOSPHOLIPID ANTIBODY SYNDROME- A DIAGNOSIS AT A LATER AGE
}

\author{
Varun $R^{1}$, Pragathee $S^{2}$
}

${ }^{1}$ Assistant Professor, Department of Anaesthesiology and Critical Care, KMCH, Coimbatore.

2 Postgraduate Student, Department of Anaesthesiology, Saveetha Medical College and Hospital, Chennai.

\section{ABSTRACT}

\section{BACKGROUND}

Antiphospholipid antibody syndrome is an autoimmune disorder involving antibody against phospholipids. It is of two types primary and secondary. We report a case of primary APLA syndrome and its management after the diagnosis was missed twice before at a younger age.

\section{KEYWORDS}

Antiphospholipid Antibody (APLA), Autoimmune Disorder, Hughes Disease, Vasoocclusive Disorder.

HOW TO CITE THIS ARTICLE: Varun R, Pragathee S. A case report of antiphospholipid antibody syndrome- a diagnosis at a later age. J. Evolution Med. Dent. Sci. 2017;6(22):1820-1822, DOI: 10.14260/Jemds/2017/400

\begin{abstract}
BACKGROUND
Antiphospholipid Antibodies (APLA) are a group of autoantibodies, all recognising various combinations of phospholipids. Primary antiphospholipid syndrome occurs in patients without clinical evidence of another autoimmune disease, whereas secondary antiphospholipid syndrome occurs in association with other autoimmune diseases. Primary APLA syndrome is an extremely rare condition. ${ }^{1}$ Our case report deals with the diagnosis of primary APLA syndrome at a later age after the opportunity to diagnose was missed twice before suggesting that APLA syndrome is still underdiagnosed and under-reported in the society.
\end{abstract}

\section{Case Report}

A 55-year-old female presented to the emergency department with complaints of inability to use the right upper limb for the past three hours. She also suffered from old cerebrovascular accident with weakness of left upper and lower limb for the past one year and is recovering. Her only son revealed that her mother had a bad obstetric history with two consecutive foetal losses. For the first conception, she had a spontaneous abortion before 10 weeks of gestation. For the second one, she had intrauterine death at term, whereas the third child was an exception though.

Clinical examination revealed she was conscious, drowsy and other higher functions were found to be normal. Other systemic examination revealed a significant fall in saturation since the arrival of the patient to ER, which was not responding to oxygen therapy. Cardiovascular system was normal and abdomen findings were nil significantly. Central nervous system examination revealed- left upper and lower limb had motor power of $3 / 5$ and right upper limb had motor power of $4 / 5$.

From the history and clinical presentation, a diagnosis of recurrent CVA was made.

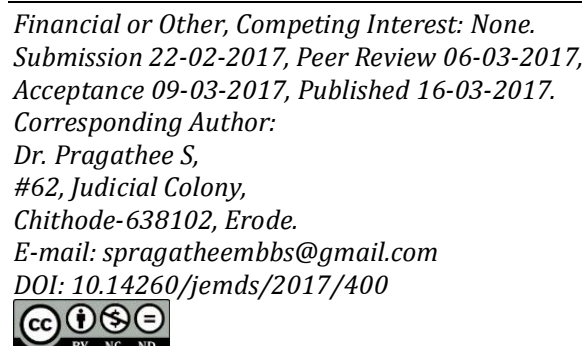

Although, her higher functions were normal and her chest findings were not significant during admission, her saturation began to fall. Even ABG which was taken could be a VBG, but the SPO2 of $22 \%$, pH- 7.18 and lactate- 10 in VBG could not be correlated. Hence, she was intubated in ER and was shifted to ICU for further management.

To rule out CVA, patient was shifted to CT and the reports showed only the old changes with infarct and gliosis in Rt. MCA territory. Hence, recurrent CVA was ruled out.

When she was received in ICU, on examining her Rt. hand she had cyanosis, cold and pulseless peripheries. Her blood sugars were always less than $70 \mathrm{mg} / \mathrm{dL}$ without any signs and symptoms of hypoglycaemia, despite the normal RFTs. She was being treated simultaneously for the same with $25 \%$ dextrose boluses and infusions.

Collectively low saturation, pulseless cold peripheries, cyanosis, and pain in the Rt. hand were correlated with ongoing ischaemia of the Rt. upper limb. Retrospectively on enquiring the nursing staff, ABG with severe acidosis, high lactate, low saturation and low sugars were all because the blood sample was collected from Rt. hand. The initial blood samples were drawn from right upper limb since the left upper limb was paralysed.

\section{Hence, Narrowing Down our Diagnosis to}

- Old CVA with Raynaud's phenomenon secondary to vasculitis (Also supported by vitiligo).

- Thromboembolic phenomenon.

Cardiologist's opinion was sought, in which echocardiography revealed inferior wall hypokinesia with a LV clot. She was started on heparin and warfarin as per cardiologist's advice to maintain target INR of 2 - 3.

Meanwhile, since her haemodynamics were stable on the other hand and metabolic parameters including blood sugars and ABG were within normal limits, she was extubated next morning in ICU. Post extubation she was stable, even vasopressors which was started in ER for haemodynamic support was tapered and stopped. Vascular surgeon's opinion was also sought for the ongoing ischaemia of the Rt. upper limb.

She was evaluated for vasculitis- aorto-occlusive disease. Doppler Aortogram showed thrombus in great vesselscommon carotids $50 \%$ occlusion on each side, Rt. subclavian artery $90 \%$ occluded which was the culprit for her symptoms in the Rt. upper limb. She was started on urokinase as infusion as per vascular surgeon's advice. 
At the Point of Evaluation, our Differential Diagnosis were-

1. Young aged stroke.

2. Large vessel vasculitis-? Takayasu's arteritis?, Kawasaki's arteritis.

3. Hypercoagulable state secondary to APLA syndrome.

Considering our DD, evaluation went towards screening for vasculitis- C-ANCA, P-ANCA, anti-DS DNA, anticardiolipin antibody, lupus anticoagulants, e NA.

For hypercoagulable state, APLA (Anticardiolipin antibody, lupus anticoagulant) protein $\mathrm{C}$ and $\mathrm{S}$ were sent. Also, it was suspected as the patient had bad obstetric history and old CVA.

Vascular surgeon's review was also sought for the possibility of thrombus aspiration. Since the vasculitis could be of autoimmune aetiology, thrombus aspiration would cause more risk of vasospasm and further aggravation of her disease than benefit. So the idea of thrombus aspiration was dropped. Vasculitis workup (C-ANCA, P-ANCA, anti-ds DNA, protein $\mathrm{C}$ and $\mathrm{S}$ ) results came as negative. But APLA- lupus anticoagulants came positive. Hence, the diagnosis of primary anti-phospholipid antibody syndrome was made and the patient was treated with steroids, urokinase infusion, anticoagulant- warfarin and other supportive measures.

Patient's right upper limb was salvaged. The patient was discharged in a haemodynamically stable state with oral prednisolone and oral warfarin and advised followup in vascular surgery outpatient department.

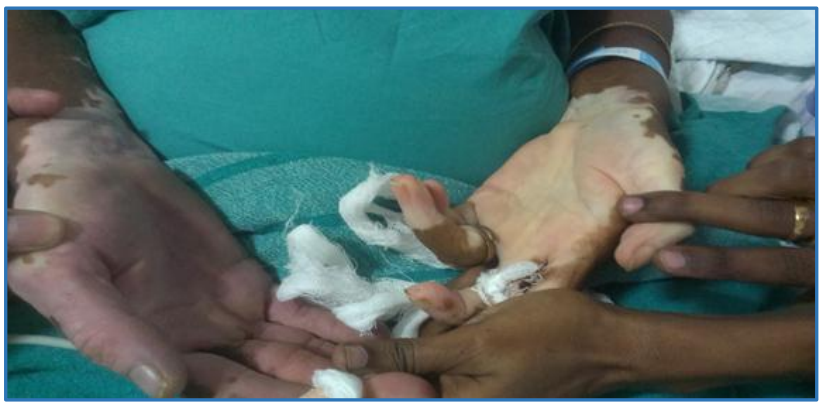

Figure 1. Shows the Cyanosis of Right Hand with Vitiligo

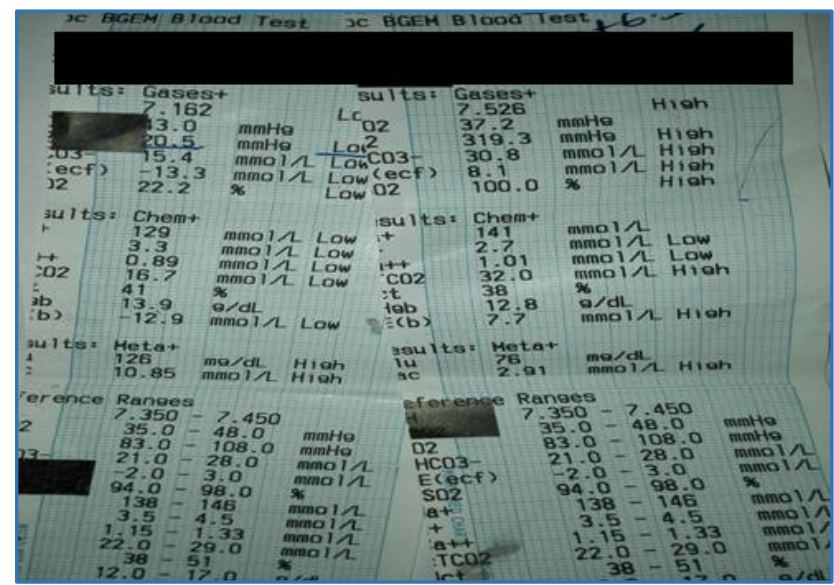

Figure 2. ABG Report on the Left from Right Radial Artery; on the Right from Left Radial Artery

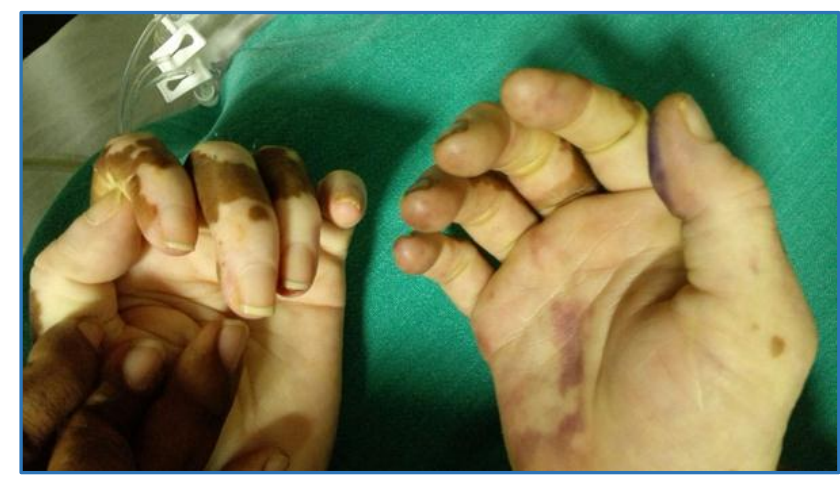

Figure 3. Shows Right Hand Cyanosis Disappeared after 3 days of Treatment

\section{DISCUSSION}

Antiphospholipid antibody syndrome or Hughes syndrome, is a systemic autoimmune disorder that involves immune mediated reactions leading to the formation of antibodies against the phospholipid membrane of the blood cells triggering the coagulation cascade and thrombosis. ${ }^{2}$ It can be either primary or secondary. ${ }^{1}$ Primary antiphospholipid syndrome occurs in patients without clinical evidence of another autoimmune disease, whereas secondary antiphospholipid syndrome occurs in association with other autoimmune diseases. The occurrence of APL antibodies associated with vasoocclusive events without any underlying disease process is termed the primary antiphospholipid antibody syndrome. ${ }^{3}$ The important antibodies that are associated with this are $\beta$ glycoprotein I, anti-cardiolipin antibodies and lupus anticoagulants. ${ }^{1}$

The APS could be inherited and rarely attributed to some environmental causes. The clinical implications of these thrombosis are arterial and venous thrombosis ranging from small vessel to the large vessels, obstetric complications leading to loss of foetuses either as abortions or IUD or preterm labour. ${ }^{2}$ Most of these clinical findings such as subclavian arterial thrombosis, serial loss of two pregnancies and a history of CVA were also present in our patient. Our patient also had intracardiac thrombi as left ventricular clot. Similar case reports of intracardiac thrombi have been reported.4,5 Premature ischaemic heart disease may occur as in our patient; however, the role of anticardiolipin antibodies in this condition is not clear. 6

This documented case of $90 \%$ thrombosis of the right subclavian artery and $50 \%$ occlusion of both common carotids in the presence of antiphospholipid antibodies fulfils the Sydney criteria for APS diagnosis, which require the presence of at least one of the clinical criteria (Vascular thrombosis and/or pregnancy morbidity) and at least one of the laboratory criteria (Detection of lupus anticoagulant, anticardiolipin or anti-beta-2-glycoprotein 1 antibodies). ${ }^{3}$ Although, the complications associated with vascular thrombosis in the present patient were serious, they seemed to appear one at a time. This is in clear contrast to catastrophic APS, where there are multiple simultaneous vascular occlusions and multiorgan involvement.

As a norm that one autoimmune disorder can be associated with other, the common autoimmune disorders associated with it are autoimmune microangiopathies including TTP, HUS, disseminated intravascular coagulopathy, Heparin induced thrombocytopenia and 
thrombosis, Systemic lupus erythematosus ${ }^{7}$ and Behcet's syndrome. This association of other autoimmune diseases occur in secondary type of APLA which is not found in our patient. In our patient ANA, ENA, anti-ds DNA, peripheral smear were found to be normal. Hence, to suspect and diagnose APS one should also have the knowledge of the other autoimmune disorders associated with it.

Finazzi et $\mathrm{al}^{8}$ (2005) randomised 109 patients with aPL and thrombosis to a target INR of $2-3$ or $3-4.5$ and followed them for a median of 3.6 years. Recurrences were $3 / 52$ $(5 \cdot 8 \%)$ in the low intensity group and $6 / 54(11 \cdot 1 \%)$ in the high intensity group. Hence, we targeted an INR of 2 - 3.

Hence, the important goals for treatment include: management of acute thrombosis with thrombolytics and heparin, maintenance therapy and thromboprophylaxis with oral warfarin to target INR 2 - 3. Oral steroid therapy with prednisolone can be instituted in the maintenance phase to prevent further flare ups. Immunoglobulins in APLA can be used in selected cases. ${ }^{9}$

\section{CONCLUSION}

To conclude, our patient with history of CVA and a bad obstetric history now presented to us with inability to use the right upper limb caused by thrombosis and occlusion of the right subclavian artery. Though the diagnosis of APLA syndrome could have been arrived in a very young age during pregnancy losses or at least during the old CVA, it was missed both the times.

Hence, this case report insists that a high index of suspicion and knowledge is needed when one evaluates the clinical finding. Henceforth, the future complications and thrombosis can be avoided with the oral anticoagulants and oral prednisolone.

\section{REFERENCES}

[1] Levine JS, Branch DW, Rauch J. The antiphospholipid syndrome. N Engl J Med 2002;346(10):752-63.

[2] Devreese K, Peerlinck K, Hoylaerts MF. Thrombotic risk assessment in the antiphospholipid syndrome requires more than the quantification of lupus anticoagulants. Blood 2010;115(4):870-8.

[3] Shrivastava A, Misra R. Apla syndrome: issues and advances. J Indian Rheumatol Assoc 2001:9.

[4] Bruce D, Bateman D, Thomas R. Left ventricular thrombi in a patient with the antiphospholipid syndrome. Br Heart J 1995;74(2):202-3.

[5] O'Neill D, Magaldi J, Dobkins D, et al. Dissolution of intracardiac mass lesions in the primary antiphospholipid antibody syndrome. Arch Intern Med 1995;155(3):325-7.

[6] Edwards T, Thomas RD, McHugh NJ. Anticardiolipin antibodies in ischaemic heart disease. Lancet 1993;342:989.

[7] Martinez-Rueda JO, Arce-Salinas CA, Kraus A, et al. Factors associated with fetal losses in severe SLE. Lupus 1996;5(2):113-9.

[8] Ferrara DE, Liu X, Espinola RG, et al. Inhibition of the thrombogenic and inflammatory properties of antiphospholipid antibodies by fluvastatin in an in vivo animal model. Arthritis Rheum 2003;48(11):3272-9.

[9] Edwards CJ, Hughes GR. Hughes syndrome (the antiphospholipid syndrome): 25 years old. Mod Rheumatol 2008;18(2):119-24. 